\title{
Clinical and genetic analyses in a patient with PAPA syndrome complicated with inflammatory bowel disease
}

\author{
H Ida ${ }^{1 *}$, Y Kunitake ${ }^{1}$, N Yoshida ${ }^{1}$, D Wakasugi ${ }^{1}$, S Kaieda ${ }^{1}$, K Mitsuyama $^{1}$, K Iwamoto ${ }^{2}$, K Fujita ${ }^{3}$, R Nishikomori ${ }^{4}$ \\ From 8th International Congress of Familial Mediterranean Fever and Systemic Autoinflammatory Diseases \\ Dresden, Germany. 30 September - 3 October 2015
}

\section{Introduction}

PAPA (pyogenic arthritis, pyoderma gangrenosum and acne) syndrome is an autoinflammatory disease linked to mutations in the PSTPIP1 gene. These mutations produce a hyper-phosphorylated PSTPIP1 protein and alter its participation in the activation of the "inflammasome".

\section{Objectives}

To elucidate the pathogenesis of PAPA syndrome, we examined the clinical status and complications and also analyzed the PSTPIP1 gene.

\section{Patients and methods}

We herein report a 23-year-old Japanese male who suffered from recurrent arthritis in his knee and ankle joints, pyoderma gangrenosum, and acne. Recently, he had experienced melena and multiple colonic ulcers had been detected by colonfiberscopy. His ulcerations resembled ulcers associated with Crohn's disease. A histological examination was then performed for the synovium of this knee joints, skin lesions of pyoderma gangrenosum, and the colon. The genomic DNA of PSTPIP1 were analysed in both the patient and his family.

\section{Results}

1) A histological analysis revealed that a large number of neutrophils had accumulated in the skin lesions; however, very few neutrophils were detected in the pathological lesions of the knee joints and colon. 2) According to a gene analysis, we detected a novel heterozygous mutation in the PSTPIP1 gene; however, his healthy father also had the same mutation, thus suggesting that this mutation of
PSTPIP1 might not be related to his phenotype. We are searching for other affected genes besides the PSTPIP1 gene for PAPA syndrome in this case.

\section{Conclusion}

We herein reported a Japanese PAPA syndrome patient who was complicated with inflammatory bowel disease. A genetic analysis suggested that this particular phenotype might not have been affected by a mutation of the PSTPIP1 gene.

\section{Authors' details}

${ }^{1}$ Kurume University School of Medicine, Department of Medicine, Kurume, Fukuoka, Japan. ${ }^{2}$ Kurume Hospital, Coloproctology Center, Kurume, Fukuoka, Japan. ${ }^{3}$ Tenri Hospital, Department of Pathology, Tenri, Nara, Japan. ${ }^{4}$ Kyoto University Graduate School of Medicine, Department of Pediatrics, Kyoto, Kyoto, Japan.

Published: 28 September 2015

doi:10.1186/1546-0096-13-S1-P148

Cite this article as: Ida et al: Clinical and genetic analyses in a patient with PAPA syndrome complicated with inflammatory bowel disease. Pediatric Rheumatology 2015 13(Suppl 1):P148. 